\title{
Correction to: Differences between bisphosphonate-related and denosumab-related osteonecrosis of the jaws: a systematic review
}

\author{
Raiza Querrer ${ }^{1}$ - Nathália Ferrare ${ }^{1}$ - Nilce Melo ${ }^{1}$ Cristine Miron Stefani ${ }^{1}$ - Paula Elaine Diniz dos Reis ${ }^{1,2}$. \\ Carla Ruffeil Moreira Mesquita ${ }^{1}$. Gabriel Alvares Borges ${ }^{1,3} \cdot$ André Ferreira Leite $^{1} \cdot$ Paulo Tadeu Figueiredo ${ }^{1}$
}

Received: 6 March 2021 / Accepted: 6 March 2021 / Published online: 18 March 2021

(C) Springer-Verlag GmbH Germany, part of Springer Nature 2021

Correction to: Supportive Care in Cancer. https://doi.org/10.1007/s00520-020-05855-6

The order of references 17, 18, 19, 20, 21, 22, 23 and 24, are reversed in the REFERENCES section and also in the citations in the text of the manuscript and in Table 1 (pages 3, 4, 5, 6, 7,8 and 9).

The correct order should be the below (references, manuscript and Table 1):

1) References

17. Moher D, Shamseer L, Clarke M, Ghersi D, Liberati A, Petticrew M, et al. PRISMA-P group. Preferred reporting items for systematic review and meta-analysis protocols (PRISMAP) 2015 statement. Systematic Reviews 2015; 4: 1-9

18. Henry D, Vadhan-Raj S, Hirsh V, von Moos R, Hungria V., Costa L, et al. Delaying skeletal-related events in a randomized phase 3 study of denosumab versus zoledronic acid in patients with advanced cancer: a analysis of data from

The online version of the original article can be found at https://doi.org/ $10.1007 / \mathrm{s} 00520-020-05855-6$

Raiza Querrer

querreer@gmail.com

Nathália Ferrare

nathaliaferrare@gmail.com

Nilce Melo

nilcesantosmelo@gmail.com

Cristine Miron Stefani

cmstefani@gmail.com

Paula Elaine Diniz dos Reis

pauladiniz@unb.br

Carla Ruffeil Moreira Mesquita

carlarm@globo.com patients with solid tumors. Support Care Cancer 2014; 22: 679-687

19. Saad F, Brown JE, Van Poznak C, Ibrahim T, Stemmer SM, Stopeck AT, et al. Incidence, risk factors, and outcomes of osteonecrosis of the jaw: integrated analysis from three blinded active-controlled phase III trials in cancer patients with bone metastases. Ann Oncol 2012; 23: 1341-1347

20. Matsui A, Kurihara J, Morishima H, Suzuki H, Sato S, Yamauchi K, et al. Medication related osteonecrosis of the jaw (MRONJ): a retrospective survey of a series of patients treated according to the AAOMS guidelines. J Oral Maxillofac Surg Med Pathol 2015; 27: 757-763

21. Baba, A, Goto, TK, Ojiri, H, Takagiwa M, Hiraga, C, Okamura, M, et al. CT imaging features of antiresorptive agent-related osteonecrosis of the jaw/medication-related
Gabriel Alvares Borges

gabriel_alvares_borges@hotmail.com

André Ferreira Leite

andreleite@unb.br

Paulo Tadeu Figueiredo

paulofigueiredo@unb.br

Health Sciences Faculty, University of Brasília, Brasilia, DF 70910-900, Brazil

2 Interdisciplinary Research Laboratory Applied to Clinical Practice in Oncology, University of Brasília, Brasilia, DF, Brazil

3 Laboratory of Oral Histopathology, Health Sciences Faculty, University of Brasilia, Brasilia, DF, Brazil 
osteonecrosis of the jaw. Dentomaxillofac Radiol 2018; 47 : 20170323

22. Klingelhoffer C, Klingelhoffer M, Müller S, Ettl T, Wahlmann U. Can dental panoramic radiographic findings serve as indicators for the development of medicationrelated osteonecrosis of the jaw? Dentomaxillofac Radiol. 2016; 45: 1-7

23. Heim N, Götz W, Kramer FJ and Faron A. Antiresorptive drug-related changes of the mandibular bone densitiy in medication-related osteonecrosis of the jaw patients. Dentomaxillofac Radiol. 2019; 48: 20190132

24. Pichardo SEC, Broek FWT, Fiocco M, AppelmanDijkstra NM, van Merkesteyn JPR. A comparison of the cone beam computed tomography (CBCT) findings in medicationrelated osteonecrosis of the jaws (MRONJ) related to denosumab vs. bisphosphonates: an observational pilot study. Oral Surg Oral Med Oral Pathol Oral Radio 2019; 19: 314968

2) MANUSCRIPT and TABLE 1 (correct order of citations according to references)

\section{PAGE 3.}

Paragraph 7 - "...The flow chart in Figure 1 illustrates the identification and selection process here described [17]. "

Paragraph 8 - "The seven included studies were published between 2011 and 2019 and conducted in the following countries: the United States [18], Canada [19], Japan [20, 21], Germany [22, 23] and the Netherlands [24]. "

Paragraph 9 - "Matsui et al. [20]..."

Paragraph 10 - "A randomized clinical trial [18]..."

Paragraph 11 - "The incidence, risk factors, and outcomes of ONJ were assessed in a study by Saad et al. [19]..."

Paragraph 12 - "Four studies described and compared the imaging aspects of BP- and DMAb-related ONJ [21-24]. In an analysis of facial panoramic radiographs, Klingelhöffer et al. [22]..."

PAGE 4 and 5:

\section{Table 1.}

Henry et al., 2014 (18).

Saad et al., 2011 (19).

Baba et al., 2018 (21).

Heim et al., 2019 (23).

Kingelhoffer et al., 2016 (22).

Matsui et al., 2015 (20).

Pichardo et al., 2019 (24).

\section{PAGE 6.}

Paragraph 2 - "Baba et al. [21] investigated ONJ via computed tomography (CT) scans..."

Paragraph 3 - "Heim et al. [23] assessed the the density of the mandibular..."
Paragraph 4 - "Pichardo et al. [24] compared the frequency of the imaging findings..."

"Fig 1 Flow chart of literature search and selection of studies, adapted from PRISMA [17]..."

\section{PAGE 7.}

Paragraph 2 - “...risk of bias of 55.5\%, 77.7\%, 75\%, 100\% and $66.6 \%$ respectively [20-24]. Randomized clinical trials resulted in a risk of bias of $80 \%$ and $66.6 \%$, respectively $[18,19] "$.

Paragraph 4 - "Even though BPs and DMAb act through different pharmacological mechanisms, three studies reported that the drugs resulted in ONJ with similar clinical characteristics $[\mathbf{1 8}, \mathbf{1 9}, \mathbf{2 4}]$. Even though one study [20] reported that patients treated with BPs presented better clinical condition,..." Paragraph 5 - "In four studies [21, 22, 23, 24], statistically significant differences between BP- and DMAb-related ONJ were observed in imaging aspects. Heim et al. [23] and Pichardo et al. [24] identified an increase in bone sequestra, cortical bone lysis and bone density in BP-related ONJ, while Baba et al. [21] and Klingelhöffer [22] noted larger bone sequestra, more frequent periosteal reactions, and mandibular canal enhancement in DMAb-related ONJ. Out of the studies that assessed imaging characteristics, three analyzed CT scans $[21,23,24]$."

\section{PAGE 8.}

Paragraph 3 - "Four of the included studies reported differences in imaging findings between BP- and DMAb-related ONJ $[\mathbf{2 1}, \mathbf{2 2}, \mathbf{2 3}, \mathbf{2 4}]$, even though they point to a divergent direction as to which is the most severe condition. Baba et al. [21] and Klingelhöffer [22] reported DMAb-related ONJ as more aggressive, while Heim et al. [23] and Pichardo et al. [24] pointed that BP-related ONJ is the most severe..."

Paragraph 4 - "It is important to note that out of those studies, one [22] compared the two conditions by assessing panoramic radiographs, while the other three $[\mathbf{2 1}, \mathbf{2 3}, \mathbf{2 4}]$ analyzed CT scans. Although both techniques are appropriate for observing imaging characteristics of $\mathrm{ONJ}, . .$.

Paragraph 5 - "...In only two studies $[18,19]$ the underlying diseases were exclusively malignant conditions. In the other studies [20, 21, 22, 23, 24], the underlying disease was either malignant, osteoporosis or rheumatoid arthritis..."

Paragraph 6 - "Another limitation concerns the lack of information on the dosage of BPs or DMAb that was administered to patients, which was described in only three studies $[18,19$, 24], as well as of data on incidence, described in four studies [20-23]. In two studies $[\mathbf{1 8}, \mathbf{1 9}]$, patients underwent similar treatment protocols, based on zoledronic acid at $4 \mathrm{mg}$ or $\mathrm{DMAb}$ at $120 \mathrm{mg}$ every four weeks... Pichardo et al. [24] reported that patients were treated with different brands..." 
Paragraph 7 - "...In some of the included studies, the comparison between BP- and DMAb-related ONJ was only a secondary observation, even among randomized clinical trials $[18-20,22] \ldots "$

PAGE 9.

Paragraph 2 - "Further studies are also advised to confirm if the time between baseline - the first use of the antiresorptive drug - and the manifestation of ONJ, is shorter in patients treated with DMAb than in patients treated with BPs, as suggested in the literature $[\mathbf{2 4}, \mathbf{2 5}, \mathbf{2 6}, \mathbf{3 3}, \mathbf{3 4}]$ '".

The original article has been corrected.

Publisher's note Springer Nature remains neutral with regard to jurisdictional claims in published maps and institutional affiliations. 